Updated version published in "Communications in Statistics: Theory and Methods",

Volume 34, Issue 7, pp 1437-1447, 2005.

\title{
New families of Copulas based on periodic functions
}

\section{Aurélien Alfonsi* Damiano Brigo**}

* Ecole Nationale des Ponts et Chaussées, 6-8 avenue Blaise Pascal, Cité Descartes, Champs sur Marne, 77455 Marne-la-vallée, France.

e-mail: alfonsi@cermics.enpc.fr

** Credit Models, Banca IMI, San Paolo IMI Group, Corso Matteotti 6 - 20121 Milano, Italy; Fax: +39027601 9324. e-mail: damiano.brigo@bancaimi.it, web page: http://www.damianobrigo.it

First version: April 1, 2003. This version: December 19, 2005

\begin{abstract}
Although there exists a large variety of copula functions, only a few are practically manageable, and often the choice in dependence modeling falls on the Gaussian copula. Further, most copulas are exchangeable, thus implying symmetric dependence. We introduce a way to construct copulas based on periodic functions. We study the two-dimensional case based on one dependence parameter and then provide a way to extend the construction to the $n$-dimensional framework. We can thus construct families of copulas in dimension $n$ and parameterized by $n-1$ parameters, implying possibly asymmetric relations. Such "periodic" copulas can be simulated easily.
\end{abstract}

Key words: Dependence Modeling, Copula Functions, Gaussian Copula, Archimedean Copula, Periodic Copula, Simulation 


\section{Introduction and Motivation}

Consider a random vector $X=\left(X_{1}, \ldots, X_{n}\right)$, and suppose that we wish to analyze the dependence between its components. The whole information on the distribution of the vector is given by the joint cumulative distribution function of $\mathrm{X}$. If $\mathbb{P}$ denotes the probability measure in our setting, such function in the point $\left(x_{1}, \ldots, x_{n}\right)$ is given by $\mathbb{P}\left(X_{1} \leq x_{1}, \ldots, X_{n} \leq x_{n}\right)$. However, this function mixes information on the dependence between the different components of the vector with information on the distribution of the single components themselves. Copula functions have been introduced in order to allow a separation between the marginal cumulative distribution functions (cdf for short) and the dependence structure. The former concerns single components, taken one at the time, and is given by the cdf's $F_{i}(x):=$ $\mathbb{P}\left(X_{i} \leq x\right), i=1, \ldots, n$, which we assume to be continuous. The latter is entirely represented by the copula function we introduce now. It is well known that $U_{1}=$ $F_{1}\left(X_{1}\right), \ldots, U_{n}=F_{n}\left(X_{n}\right)$ are uniformly distributed random variables on $[0,1]$. The joint cumulative distribution function of $\left(U_{1}, \ldots, U_{n}\right)$, that we denote by

$$
C\left(u_{1}, \ldots, u_{n}\right)=\mathbb{P}\left(U_{1} \leq u_{1}, \ldots, U_{n} \leq u_{n}\right)
$$

is called the copula function of $\left(X_{1}, \ldots, X_{n}\right)$ and has the following link with the multivariate cdf:

$$
\mathbb{P}\left(X_{1} \leq x_{1}, \ldots, X_{n} \leq x_{n}\right)=C\left(\mathbb{P}\left(X_{1} \leq x_{1}\right), \ldots, \mathbb{P}\left(X_{n} \leq x_{n}\right)\right)
$$

One can easily check that a copula has the following properties:

1. $C\left(u_{1}, . ., u_{i-1}, 0, u_{i+1}, . ., u_{n}\right)=0$

2. $C\left(1, . ., 1, u_{k}, 1, . ., 1\right)=u_{k}$

3. $\partial_{u_{1} \ldots u_{n}} C$ is a positive measure in the sense of Schwartz distributions. This means concretely that for any hypercube $H=\left[a_{1}, b_{1}\right] \times \ldots \times\left[a_{n}, b_{n}\right] \subset[0,1]^{n}$,

$$
\mathbb{P}\left[\left(U_{1}, . ., U_{n}\right) \in H\right] \geq 0
$$

When $n=2$, this can be written as

$$
C\left(b_{1}, b_{2}\right)-C\left(a_{1}, b_{2}\right)-C\left(b_{1}, a_{2}\right)+C\left(a_{1}, a_{2}\right) \geq 0 \text {. }
$$


Conversely, one can show that any function that satisfies these three conditions can be viewed as the joint cdf of a vector of uniform variables on $[0,1]$ and is thus a copula. This is known as Sklar's theorem, see for example Joe (1997) or Nelsen (1999).

In the following, the expression "simulating a copula $C$ " will denote the simulation of a random vector of uniform variables $\left(U_{1}, . ., U_{n}\right)$ on $[0,1]$ whose joint cdf is $C$.

Among the different ways to define specific copula functions, there are following two. The first one consists in seeking functions $C$ satisfying the three above properties. Archimedean copulas are an example of this approach. Indeed, Archimedean copulas come from the remark that if $\varphi$ is a convex decreasing function such that $\varphi(1)=0$, then

$$
C\left(u_{1}, . ., u_{n}\right)=\mathbf{1}_{\left\{\varphi\left(u_{1}\right)+. .+\varphi\left(u_{n}\right) \leq \varphi(0)\right\}} \varphi^{-1}\left(\varphi\left(u_{1}\right)+. .+\varphi\left(u_{n}\right)\right)
$$

has the above three properties and is thus a copula. Therefore, by specifying families of decreasing convex functions that vanish in 1 we specify families of copulas (e.g. Gumbel, Joe, Frank...), see Bouyé et al. (2000), Nelsen (1999) and Joe (1997).

The second method consists in working directly with joint cdf's $F\left(x_{1}, \ldots, x_{n}\right)$ and the related marginal cdf's $F_{i}$. The associated copula is then defined as $F\left(F_{1}^{-1}\left(u_{1}\right), \ldots, F_{n}^{-1}\left(u_{n}\right)\right)$. Even if this method does not always lead to analytically tractable copulas, it can provide us copulas that are easy to simulate. Indeed, the main example of this kind of construction is the well known fundamental family of Gaussian copulas. A Gaussian copula is defined as the copula of a joint Gaussian random vector $X$ with standard Gaussian marginals and correlation matrix $\rho$, and is thus given by $N_{\rho}\left(N^{-1}\left(u_{1}\right), \ldots, N^{-1}\left(u_{n}\right)\right)$ where $N$ is the cdf of a standard normal variable and $N_{\rho}$ is the joint cdf of $X$. This copula cannot be computed explicitly. The simulation is however straightforward: it is sufficient to consider $\left(N\left(X_{1}\right), \ldots, N\left(X_{n}\right)\right)$ where $X=\left(X_{1}, \ldots, X_{n}\right)$ is a Gaussian vector with correlation $\rho$ that can be easily simulated by resorting to a standard Gaussian simulator and to a Cholesky decomposition of . A similar approach leads to Student's copula (see Bouyé et al. (2000). and Genz and Bretz (2002)

A possible major drawback of Archimedean and Gaussian copulas is that they are $n$-exchangeable ( $n$-symmetric): if $\sigma$ is a permutation on $\{1, \ldots, n\}$, we have $C\left(u_{1}, . ., u_{n}\right)=C\left(u_{\sigma(1)}, . ., u_{\sigma(n)}\right)$. Let us extend this notion by the following definition. 
Definition 1.1. (k-exchangeability). Let us consider a copula $C$ that is the cdf of the random vector $\left(U_{1}, . ., U_{n}\right)$. We will say that the copula $C$ is $k$-exchangeable $(2 \leq k \leq n)$ if, for any $1 \leq i_{1}<i_{2}<. .<i_{k} \leq n$ and any permutation $\sigma$ on $\{1, \ldots, k\},\left(U_{i_{1}}, . ., U_{i_{k}}\right)$ and $\left(U_{i_{\sigma(1)}}, . ., U_{i_{\sigma(k)}}\right)$ have the same law.

It is clear, with this definition, that a $k^{\prime}$-exchangeable copula is also a $k$-exchangeable copula whenever $k^{\prime}>k$. In the two-dimensional case we resort directly to the term "exchangeable" rather than "2-exchangeable". When dealing with defaultable bonds as in Jouanin and al. (2001), n-exchangeable copulas cannot model situations where the dependence is asymmetric and based on the assets themselves. With 2-echangeable copulas (such as for example Gaussian or Archimedean copulas), we cannot model asymmetric relations featuring a first entity that influences a second one more than the latter influences the former.

In recent years, copula functions have received a great deal of attention, see for example the papers of Genz and Bretz (2002), Hürlimann (2002, 2003), Juri and Wüthrich (2002), Nelsen et al. (2001), Wei and Hu (2002), and the books of Joe (1997) and Nelsen (1999). For financial and insurance applications, recent applications on copulas include for example Bouyé et al. (2000), Cherubini et al. (2002), Embrechts et al. (2001), Jouanin et al. (2001), Klugman and Parsa (1999), Prampolini (2003), and Schönbucher and Schubert (2001).

In this paper, we will build new families of copulas based on the first approach, using periodic functions following Alfonsi (2002). We first begin to work in the two-dimensional case, obtaining a one-parameter copula, and then give a way to extend the result to the $n$-dimensional case with $n>2$, getting a family with $n-1$ parameters. Finally, we explain how such copulas can be simulated.

\section{Construction of copulas based on periodic func- tions}

\subsection{The construction of families in two dimensions}

We begin by defining our new copula functions for bivariate dependence, i.e. for possible dependence structures between two random variables. It is helpful to first recall three particular "limit" copulas. The "middle" one, which is typically denoted 
by $C^{\perp}$, is the copula obtained when $U_{1}$ and $U_{2}$ are independent uniform variables on $[0,1]$, that is:

$$
C^{\perp}\left(u_{1}, u_{2}\right):=u_{1} u_{2}
$$

The two other "limit" copulas, denoted by $C_{F}^{-}$and $C_{F}^{+}$respectively, are the two Frechet bounds of the convex subset of copulas:

$$
C_{F}^{+}\left(u_{1}, u_{2}\right)=\min \left(u_{1}, u_{2}\right), \quad C_{F}^{-}\left(u_{1}, u_{2}\right)=\left(u_{1}+u_{2}-1\right)^{+}
$$

where $x^{+}=\max (x, 0)$ denotes the positive part operator. Naming $U$ a uniform random variable on $[0,1], C_{F}^{+}$can be obtained as the copula of $(U, U)$ and corresponds obviously to perfect positive dependence, whereas $C_{F}^{-}$is obtained as the copula of $(U, 1-U)$ and describes total negative dependence. Moreover, for any copula $C$, we have

$$
C_{F}^{-}\left(u_{1}, u_{2}\right) \leq C\left(u_{1}, u_{2}\right) \leq C_{F}^{+}\left(u_{1}, u_{2}\right), \forall\left(u_{1}, u_{2}\right) \in[0,1]^{2} .
$$

Recall the above characterization of a copula function for the bivariate case: the function $C$ defined on $[0,1]^{2}$ is a copula if and only if i) $C\left(u_{1}, 0\right)=0$ and $C\left(0, u_{2}\right)=u_{2}$, ii) $C\left(u_{1}, 1\right)=u_{1}$ and $C\left(1, u_{2}\right)=u_{2}$, and iii) $\frac{\partial^{2} C}{\partial u_{1} \partial u_{2}}$ is a positive measure in the sense of Schwartz distributions.

We will say in the following that a copula admits a density when $\frac{\partial^{2} C}{\partial u_{1} \partial u_{2}}=c\left(u_{1}, u_{2}\right)$ exists in the ordinary sense. In this paper we propose copulas that have a density that can be written in the form

$$
c\left(u_{1}, u_{2}\right)=\tilde{c}\left(u_{1}+u_{2}\right) \quad\left(\operatorname{resp} . c\left(u_{1}, u_{2}\right)=\tilde{c}\left(u_{1}-u_{2}\right)\right)
$$

for a function $\tilde{c}: \mathbb{R} \rightarrow \mathbb{R}$. To satisfy properties i), ii) and iii), $\tilde{c}$ must be nonnegative and verify:

$$
\begin{gathered}
\int_{0}^{u_{1}} \int_{0}^{1} \tilde{c}\left(x_{1} \pm x_{2}\right) d x_{1} d x_{2}=u_{1}, \forall u_{1} \in[0,1] \\
\int_{0}^{1} \int_{0}^{u_{2}} \tilde{c}\left(x_{1} \pm x_{2}\right) d x_{1} d x_{2}=u_{2}, \forall u_{2} \in[0,1] .
\end{gathered}
$$

Differentiating with respect to $u_{1}$ and $u_{2}$ respectively, we obtain

$$
\begin{aligned}
& \int_{0}^{1} \tilde{c}\left(u_{1} \pm x_{2}\right) d x_{2}=1, \quad \forall u_{1} \in[0,1] \\
& \int_{0}^{1} \tilde{c}\left(x_{1} \pm u_{2}\right) d x_{1}=1, \quad \forall u_{2} \in[0,1] .
\end{aligned}
$$


Differentiating further the first relation, since $\int_{0}^{1} \tilde{c}\left(u_{1}+x_{2}\right) d x_{2}=\int_{u_{1}}^{u_{1}+1} \tilde{c}\left(x_{2}\right) d x_{2}$ (resp. $\left.\int_{0}^{1} \tilde{c}\left(u_{1}-x_{2}\right) d x_{2}=\int_{u_{1}-1}^{u_{1}} \tilde{c}\left(x_{2}\right) d x_{2}\right)$, we obtain:

$$
\tilde{c}\left(u_{1}+1\right)=\tilde{c}\left(u_{1}\right) \forall u_{1} \in[0,1], \quad\left(\operatorname{resp} . \tilde{c}\left(u_{1}-1\right)=\tilde{c}\left(u_{1}\right) \forall u_{1} \in[0,1]\right) .
$$

Thus, a consequence of requiring $c\left(u_{1}, u_{2}\right):=\tilde{c}\left(u_{1} \pm u_{2}\right)$ to be the density of a copula is that $\tilde{c}$ has to be 1 -periodic (at least on $[-1,2]$, but its value outside this interval is irrelevant) and that $\int_{0}^{1} \tilde{c}(u) d u=1$. Conversely, it is easy to see that if $\tilde{c}$ is a nonnegative 1-periodic function such that $\int_{0}^{1} \tilde{c}(u) d u=1$, then

$$
\widetilde{C}^{-}\left(u_{1}, u_{2}\right):=\int_{0}^{u_{1}} \int_{0}^{u_{2}} \tilde{c}\left(x_{1}+x_{2}\right) d x_{1} d x_{2} \quad\left(\operatorname{resp} . \widetilde{C}^{+}\left(u_{1}, u_{2}\right):=\int_{0}^{u_{1}} \int_{0}^{u_{2}} \tilde{c}\left(x_{1}-x_{2}\right) d x_{1} d x_{2}\right)
$$

satisfies conditions i), ii) and iii), and so is a copula function that we call, with a slight abuse of language, periodic copula. We note here that copulas obtained with these densities form a convex set since a convex combination of 1-periodic nonnegative functions satisfying $\int_{0}^{1} \tilde{c}(u) d u=1$ is also a 1-periodic nonnegative function with integral 1 on a period. Notice further that the use of the "-" and "+" signs appears to be counterintuitive (one would exchange the above signs), but there is a reason for this that will be clarified later on.

At times, rather than characterizing copulas through their densities, it is preferable to have a direct characterization of the copula itself. To characterize periodic copulas without explicitly referring to their densities, denote by $\varphi$ the primitive of $\tilde{c}$ that vanishes at 0 , and set $\Phi(x):=\int_{0}^{x} \varphi(u) d u$, so that $\Phi$ is a double primitive of $\tilde{c}$. We can then rewrite the above periodic copula as follows:

$$
\begin{aligned}
& \widetilde{C}^{-}\left(u_{1}, u_{2}\right)=\int_{0}^{u_{1}} \int_{0}^{u_{2}} \tilde{c}\left(x_{1}+x_{2}\right) d x_{1} d x_{2}=\Phi\left(u_{1}+u_{2}\right)-\Phi\left(u_{1}\right)-\Phi\left(u_{2}\right), \\
& \widetilde{C}^{+}\left(u_{1}, u_{2}\right)=\int_{0}^{u_{1}} \int_{0}^{u_{2}} \tilde{c}\left(x_{1}-x_{2}\right) d x_{1} d x_{2}=\Phi\left(u_{1}\right)+\Phi\left(-u_{2}\right)-\Phi\left(u_{1}-u_{2}\right)
\end{aligned}
$$

and we see that the first copula is always exchangeable (symmetric), in that $\widetilde{C}^{-}\left(u_{1}, u_{2}\right)=$ $\widetilde{C}^{-}\left(u_{2}, u_{1}\right)$, whereas the second one can be non symmetric if $\Phi$ is not par, i.e. if $\Phi(-x) \neq \Phi(x)$ for some $x$. We have thus characterized our periodic copulas in terms of double primitives $\Phi$ of periodic functions $\tilde{c}$.

A first example of such a function which arises naturally is $\tilde{c}(x)=1+\sin (2 \pi x+\varphi)$ where $\varphi$ is a parameter that we can take in $[0,2 \pi)$. It gives respectively the following families of copulas: 
- $\widetilde{C}^{-}\left(u_{1}, u_{2}\right)=u_{1} u_{2}+\left(\sin \left(2 \pi u_{1}+\varphi\right)-\sin (\varphi)-\sin \left(2 \pi\left(u_{1}+u_{2}\right)+\varphi\right)+\sin \left(2 \pi u_{2}+\right.\right.$ $\varphi)) /(2 \pi)^{2}$

- $\widetilde{C}^{+}\left(u_{1}, u_{2}\right)=u_{1} u_{2}+\left(\sin (\varphi)-\sin \left(2 \pi u_{1}+\varphi\right)+\sin \left(2 \pi\left(u_{1}-u_{2}\right)+\varphi\right)-\sin \left(-2 \pi u_{2}+\right.\right.$ $\varphi)) /(2 \pi)^{2}$.

These copulas, however, cannot model strong positive or negative dependence, since these copulas cannot approach neither $C_{F}^{-}$nor $C_{F}^{+}$. On the contrary, it might be interesting to have a family of copulas which attains the copulas $C_{F}^{+}, C^{\perp}$ and $C_{F}^{-}$ as limit cases in order to be able to describe a large range of dependence structures. By expressing copulas by means of $\frac{\partial^{2} C}{\partial u_{1} \partial u_{2}}$, attaining $C_{F}^{+}, C_{F}^{-}$and $C^{\perp}$ amounts to attaining

$$
\mu^{+}=\delta_{x_{1}}\left(d x_{2}\right) \otimes d x_{1}, \quad \mu^{-}=\delta_{1-x_{1}}\left(d x_{2}\right) \otimes d x_{1}, \quad c^{\perp}=1 .
$$

with the copula density. Since $\frac{\partial^{2} C^{\perp}}{\partial u_{1} \partial u_{2}}$ exists in the ordinary sense of differentiation, it has a density $c^{\perp}=1$ that corresponds to the Lebesgue measure on the square $[0,1]^{2}, \mu^{\perp}=d x_{1} \otimes d x_{2}$. Instead, $\mu^{+}$and $\mu^{-}$are to be interpreted in the generalized sense. Moreover, $\mu^{+}$and $\mu^{-}$charge only the diagonals of the square $[0,1]^{2}$, i.e. $\Delta^{+}=\{(x, x), x \in[0,1]\}$ and $\Delta^{-}=\{(x, 1-x), x \in[0,1]\}$ respectively. The idea is then to find a family of periodic functions $\tilde{c}_{\gamma}$ indexed by a parameter $\gamma$ and such that the density $\tilde{c}_{\gamma}\left(x_{1}-x_{2}\right)$ (resp. $\tilde{c}_{\gamma}\left(x_{1}+x_{2}\right)$ ) concentrates on $\Delta^{+}\left(\right.$resp. $\left.\Delta^{-}\right)$for some values of $\gamma$. Thus, if we define the piecewise 1 -periodic function $\tilde{c}_{\gamma}$ for $0<\gamma \leq \frac{1}{2}$ by

$$
\tilde{c}_{\gamma}(x):=\frac{1}{2 \gamma}\left(\mathbf{1}_{[0, \gamma]}(x)+\mathbf{1}_{(1-\gamma, 1)}(x)\right) \text { for } x \in[0,1)
$$

we see that the family of densities defined as $c_{\gamma}^{+}\left(x_{1}, x_{2}\right):=\tilde{c}_{\gamma}\left(x_{1}-x_{2}\right)$ verifies:

$$
c_{1 / 2}^{+}=c^{\perp}, \quad c_{\gamma}^{+}\left(x_{1}, x_{2}\right) d x_{1} d x_{2} \underset{\gamma \rightarrow 0}{\stackrel{\mathcal{D}}{\longrightarrow}} \mu^{+}
$$

$\stackrel{\mathcal{D}}{\rightarrow}$ denoting convergence in distribution. The corresponding convergence in law for random variables is denoted by $\mathcal{L}$. To calculate the associated copula $C_{\gamma}^{+}:=\widetilde{C}^{+}$, it is best to try a drawing and see that its value in $\left(u_{1}, u_{2}\right)$ is the area in the intersection of the rectangle delimited by $(0,0)$ and $\left(u_{1}, u_{2}\right)$ with $\left\{\left(x_{1}, x_{2}\right) \in[0,1]^{2},-\gamma \leq x_{1}-x_{2} \leq \gamma\right.$ or $x_{1}-x_{2} \leq \gamma-1$ or $\left.x_{1}-x_{2} \geq 1-\gamma\right\}$. We obtain, for $u_{1} \leq u_{2}$ which is not restrictive since $C_{\gamma}^{+}\left(u_{1}, u_{2}\right)=C_{\gamma}^{+}\left(u_{2}, u_{1}\right)$,

$$
\begin{aligned}
C_{\gamma}^{+}\left(u_{1}, u_{2}\right)= & \frac{1}{2 \gamma}\left[u_{1} u_{2}+\frac{1}{2}\left[-\left(\left(u_{2}-u_{1}-\gamma\right)^{+}+\left(u_{2}-\gamma\right)^{+}\right)\left(\min \left(u_{1}, u_{2}-\gamma\right)\right)^{+}-\left(\left(u_{1}-\gamma\right)^{+}\right)^{2}\right.\right. \\
& \left.\left.+\left(\left(u_{2}-1+\gamma\right)^{+}+\left(u_{2}-1+\gamma-u_{1}\right)^{+}\right) \cdot\left(\min \left(u_{1}, u_{2}-1+\gamma\right)\right)^{+}+\left(\left(u_{1}-1+\gamma\right)^{+}\right)^{2}\right]\right]
\end{aligned}
$$


In order to obtain a family that reaches $C_{F}^{-}$instead, we use the other family, precisely $c_{\gamma}^{-}\left(x_{1}, x_{2}\right)=\tilde{c}_{\gamma}\left(x_{1}+x_{2}\right)$. We obtain

$$
c_{1 / 2}^{-}=c^{\perp}, \quad c_{\gamma}^{-}\left(x_{1}, x_{2}\right) d x_{1} d x_{2} \underset{\gamma \rightarrow 0}{\stackrel{\mathcal{D}}{\longrightarrow}} \mu^{-} .
$$

Fortunately, a simple geometric remark links the related copula $C_{\gamma}^{-}$to $C_{\gamma}^{+}$, thus avoiding a new calculation:

$$
C_{\gamma}^{-}\left(u_{1}, u_{2}\right)=u_{2}-C_{\gamma}^{+}\left(1-u_{1}, u_{2}\right)
$$

Thus, with this method, we have obtained a family of copula quite "exhaustive" going from $C_{F}^{-}$to $C_{F}^{+}$and taking the in-between value $C^{\perp}$. Incidentally, we see now why we chose to name $\widetilde{C}^{+}$the copula coming from $\tilde{c}\left(x_{1}-x_{2}\right)$ and $\widetilde{C}^{-}$the copula coming from $\tilde{c}\left(x_{1}+x_{2}\right)$ : this is done because in our case the former attains $C_{F}^{+}$and the latter $C_{F}^{-}$.

At times it can be handy to have a single number measuring some stylized aspects of a given copula. The Spearman's rho is such a number and is a well known measure of concordance, see for example Embrechts et al. (2001). When defined in terms of copula functions, it is given by the following integral in the copula density $c: \rho:=$ $12 \int_{0}^{1} \int_{0}^{1} u_{1} u_{2} c\left(u_{1}, u_{2}\right) d u_{1} d u_{2}-3$. We obtain for the $C_{\gamma}^{+}$and $C_{\gamma}^{-}$copulas respectively,

$$
\rho_{\gamma}^{+}=(2 \gamma-1)(\gamma-1), \quad \rho_{\gamma}^{-}=(1-2 \gamma)(\gamma-1)
$$

An interesting remark concerns the construction of non-exchangeable (non-symmetric) copulas $\left(C\left(u_{1}, u_{2}\right) \neq C\left(u_{2}, u_{1}\right)\right)$ through this method. This can be relevant for example in credit risk when modelling the default dependence between two firms with asymmetric relations. One may have a first firm depending more on a second one than the latter depends on the former. This could be the case of a little firm that provides goods to a large one. A default of the large company could induce a dramatic effect on the smaller one, whereas a default of the small firm could have little relevance to the large one. Notice that, in this respect, Archimedean and Gaussian copulas only provide symmetric relations between the two firms defaults.

In order to provide an example of non-exchangeable copula obtained from our family, we see from (2) that our only chance is to select a periodic function $\tilde{c}$ whose double primitive $\Phi$ is not par and then take the related $\widetilde{C}^{+}$. The simplest such function is $\tilde{c}:=\bar{c}_{\gamma}$ defined, for $\gamma \in[0,1]$, as

$$
\bar{c}_{\gamma}(x):=(1 / \gamma) \mathbf{1}_{[0, \gamma]}(x), \text { for } x \in[0,1)
$$


We have then $\bar{c}_{\gamma}(x)=\tilde{c}_{\frac{\gamma}{2}}\left(x-\frac{\gamma}{2}\right)$. Thus, we obtain the following copula $\bar{C}_{\gamma}^{+}:=\widetilde{C}^{+}$ associated with the density $\tilde{c}\left(x_{1}-x_{2}\right):=\bar{c}_{\gamma}\left(x_{1}-x_{2}\right)$ :

$$
\begin{aligned}
\bar{C}_{\gamma}^{+}\left(u_{1}, u_{2}\right) & =\int_{0}^{u_{1}} \int_{0}^{u_{2}} \bar{c}_{\gamma}\left(x_{1}-x_{2}\right) d x_{1} d x_{2}=\int_{0}^{u_{1}} \int_{0}^{u_{2}} \tilde{c}_{\gamma / 2}\left(x_{1}-x_{2}-\gamma / 2\right) d x_{1} d x_{2} \\
& =\int_{0}^{u_{1}} \int_{\frac{\gamma}{2}}^{u_{2}+\gamma / 2} \tilde{c}_{\gamma / 2}\left(x_{1}-x_{2}\right) d x_{1} d x_{2} \\
& =C_{\gamma / 2}^{+}\left(u_{1}, \min \left(u_{2}+\gamma / 2,1\right)\right)-C_{\gamma / 2}^{+}\left(u_{1}, \gamma / 2\right)+C_{\gamma / 2}^{+}\left(u_{1},\left(u_{2}+\gamma / 2-1\right)^{+}\right)
\end{aligned}
$$

With this asymmetric periodic copula we still have good asymptotic properties, in that $\bar{C}_{1}^{+}=C^{\perp}$ and $\bar{C}_{\gamma}^{+} \rightarrow C_{F}^{+}$when $\gamma \rightarrow 0$. Calculating the Spearman's rho, we find again $\rho\left(\bar{C}_{\gamma}^{+}\right)=(2 \gamma-1)(\gamma-1)$ for $\gamma \in[0,1]$. Somehow surprisingly, $\rho\left(\bar{C}_{\gamma}^{+}\right)$ takes negative value between $\gamma=1 / 2$ and $\gamma=1$, and vanishes at $1 / 2$ for a copula different from $C^{\perp}$. To obtain a (symmetric) family that reaches $C_{F}^{-}$we need consider $\bar{C}_{\gamma}^{-}\left(u_{1}, u_{2}\right):=\int_{0}^{u_{1}} \int_{0}^{u_{2}} \bar{c}_{\gamma}\left(x_{1}+x_{2}\right) d x_{1} d x_{2}$. We get

$$
\bar{C}_{\gamma}^{-}\left(u_{1}, u_{2}\right)=C_{\gamma / 2}^{-}\left(u_{1},\left(u_{2}-\gamma / 2\right)^{+}\right)+C_{\gamma / 2}^{-}\left(u_{1}, 1-\left(\gamma / 2-u_{2}\right)^{+}\right)-C_{\gamma / 2}^{-}\left(u_{1}, 1-\gamma / 2\right)
$$

and can show, with a trivial change of variable, that $\rho\left(\bar{C}_{\gamma}^{-}\right)=-\rho\left(\bar{C}_{\gamma}^{+}\right)=-(2 \gamma-$ $1)(\gamma-1)$. Thus, if we wish to describe a negative asymmetric dependence, it is best to use $C_{\gamma}^{+}$with $1 / 2<\gamma<1$. However, we point out that we cannot describe negative dependence with an asymmetric copula attaining $C_{F}^{-}$.

Another interesting synthetic quantity concerning copulas is the upper-tail dependence. This indicator is defined as

$$
\lambda:=\lim _{u \rightarrow 0} \frac{1}{u} \int_{1-u}^{1} \int_{1-u}^{1} c\left(x_{1}, x_{2}\right) d x_{1} d x_{2}
$$

when the copula has a density $c$. Let us consider the general periodic case, where as before $\tilde{c}$ is a nonnegative 1-periodic function such that $\int_{0}^{1} \tilde{c}(u) d u=1$, and $\varphi$ is its primitive that vanishes at 0 . Using the periodicity, we have $\frac{1}{u} \int_{1-u}^{1} \int_{1-u}^{1} c\left(x_{1} \pm\right.$ $\left.x_{2}\right) d x_{1} d x_{2}=\frac{1}{u} \int_{-u}^{0} \int_{-u}^{0} c\left(x_{1} \pm x_{2}\right) d x_{1} d x_{2}=\frac{1}{u} \int_{-u}^{0} \pm\left(\varphi\left(x_{1}\right)-\varphi\left(x_{1} \mp u\right)\right) d x_{1} \underset{u \rightarrow 0}{\rightarrow} 0$ since $\lim _{u \rightarrow 0} \frac{1}{u} \int_{-u}^{0} \varphi\left(x_{1}\right) d x_{1}=\lim _{u \rightarrow 0} \frac{1}{u} \int_{0}^{u} \varphi\left(x_{1}\right) d x_{1}=\varphi(0)=0$. Thus, periodic copulas have no upper-tail dependence. However, if one wishes to obtain a copula with an upper-tail dependence equal to $\lambda>0$, it is still possible to consider the convex combination $(1-\lambda) C+\lambda C_{F}^{+}$where $C$ is a preferred periodic copula. This convex combination can be simulated easily when one knows how to simulate the basic $C$, as we do for the periodic copulas (with invertible $\varphi$, i.e. with a strictly positive periodic function $\tilde{c})$ we introduced here. 


\subsection{A smooth family that reaches $C_{F}^{-}, C^{\perp}$ and $C_{F}^{+}$}

A drawback of the families $C_{\gamma}^{+}$and $C_{\gamma}^{-}$is that these copulas are constant on some intervals and comes from the 0-1 nature of the density, and more precisely from the existence of a domain (with positive measure) where the density vanishes. This causes problems, especially when in need of simulating the copula. In order to avoid this drawback, the idea is then to replace $\gamma$ with a random variable $\Gamma$ and then take the expectation of $\tilde{c}_{\Gamma}$, using the convexity of the subset of the periodic copulas. Indeed, if $\Gamma \sim p$ where $p$ is the density of a probability measure on $[0,1 / 2]$ such that $p(\gamma)>0 \forall \gamma$, then $\widehat{c}_{p}(x):=E\left[\tilde{c}_{\Gamma}(x)\right]=\int_{0}^{\frac{1}{2}} \tilde{c}_{\gamma}(x) p(\gamma) d \gamma$ is a positive 1-periodic function. Thus, if we have a family of random variables $\left(\Gamma_{\alpha}\right)_{\alpha \geq 0}$ concentrated on $[0,1 / 2]$ with densities $\left\{p_{\alpha}, \alpha \in\right] 0,+\infty[\}$ on $[0,1 / 2]$ and such that $\Gamma_{\alpha} \underset{\alpha \rightarrow 0}{\stackrel{\mathcal{L}}{\longrightarrow}} 1 / 2$ and $\Gamma_{\alpha} \underset{\alpha \rightarrow \infty}{\stackrel{\mathcal{L}}{\longrightarrow}} 0$, we can define

$$
\widehat{C}_{\alpha}^{+}:=E\left[C_{\Gamma_{\alpha}}^{+}\right]=\int_{0}^{\frac{1}{2}} C_{\gamma}^{+} p_{\alpha}(\gamma) d \gamma, \quad \widehat{C}_{\alpha}^{-}:=E\left[C_{\Gamma_{\alpha}}^{-}\right]=\int_{0}^{\frac{1}{2}} C_{\gamma}^{-} p_{\alpha}(\gamma) d \gamma
$$

(that correspond respectively to the periodic densities $\tilde{c}\left(x_{1}-x_{2}\right)=\widehat{c}_{p_{\alpha}}\left(x_{1}-x_{2}\right)$ and $\left.\tilde{c}\left(x_{1}+x_{2}\right)=\widehat{c}_{p_{\alpha}}\left(x_{1}+x_{2}\right)\right)$. We have obtained a family of copulas with good asymptotic properties, in that

$$
\widehat{C}_{\alpha}^{+} \underset{\alpha \rightarrow 0}{\rightarrow} C^{\perp}, \quad \widehat{C}_{\alpha}^{+} \underset{\alpha \rightarrow+\infty}{\rightarrow} C_{F}^{+}, \quad \widehat{C}_{\alpha}^{-} \underset{\alpha \rightarrow 0}{\rightarrow} C^{\perp}, \text { and } \widehat{C}_{\alpha}^{-} \underset{\alpha \rightarrow+\infty}{\rightarrow} C_{F}^{-} .
$$

We can build easily a random variable with suitable density on $\left[0, \frac{1}{2}\right]$ by transforming a uniform variable $U$ on $[0,1]$ according to a homeomorphism. Indeed, consider $\Gamma_{\alpha}:=$ $\left.\frac{1}{2} U^{\alpha}, \alpha \in\right] 0,+\infty\left[\right.$, so that we get a family of densities $p_{\alpha}$ on $\left[0, \frac{1}{2}\right]$ that feature the desired asymptotic properties in 0 and $+\infty$ and are immediately computed:

$$
p_{\alpha}(u)=\left(2^{\frac{1}{\alpha}} / \alpha\right) u^{\frac{1-\alpha}{\alpha}}
$$

The calculation of $C_{\alpha}^{+}$does not present difficulties either. We first calculate the periodic function $\widehat{c}_{\alpha}:=\widehat{c}_{p_{\alpha}}$, obtaining

$$
\begin{aligned}
\widehat{c}_{\alpha}(x) & =\frac{1}{1-\alpha}\left[1-(2 x)^{\frac{1-\alpha}{\alpha}}\right], \alpha \neq 1 \\
\widehat{c}_{1}(x) & =-\ln (2 x)
\end{aligned}
$$

for $0 \leq x \leq 1 / 2$, and $\widehat{c}_{\alpha}(x)=\widehat{c}_{\alpha}(1-x)$ for $1 / 2 \leq x \leq 1$, since the same property holds for the basic $\tilde{c}_{\gamma}$ 's. Let us compute the primitive $\psi^{\alpha}$ of $\widehat{c}_{\alpha}$ that vanishes at $x=0$. We obtain, for $0 \leq x \leq 1 / 2$ :

$$
\psi_{\alpha}(x)=\frac{1}{2(1-\alpha)}\left[2 x-\alpha(2 x)^{\frac{1}{\alpha}}\right], \alpha \neq 1, \quad \psi_{1}(x)=x-x \ln (2 x) .
$$


Using the symmetry property $\widehat{c}_{\alpha}(x)=\widehat{c}_{\alpha}(1-x)$ we obtain, for $1 / 2 \leq x \leq 1, \psi_{\alpha}(x)=$ $\psi_{\alpha}(1 / 2)+\left(\psi_{\alpha}(1 / 2)-\psi_{\alpha}(1-x)\right)=1-\psi_{\alpha}(1-x)$, since $\psi_{\alpha}(1 / 2)=1 / 2$. Instead, for $x \in[-1,0]$ we use the periodicity of $\widehat{c}$ to get $\psi_{\alpha}(x)=-\psi_{\alpha}(-x)$. To proceed further, we need to know also the primitive $\Psi_{\alpha}$ of $\psi_{\alpha}$, i.e. the double primitive of $\widehat{c}_{\alpha}$. We find, for $x \in\left[0, \frac{1}{2}\right]$ :

$$
\Psi_{\alpha}(x)=\frac{1}{2(1-\alpha)}\left[x^{2}-\frac{\alpha^{2}}{\alpha+1} 2^{\frac{1}{\alpha}} x^{\frac{1+\alpha}{\alpha}}\right], \alpha \neq 1, \quad \Psi_{1}(x)=\frac{3}{4} x^{2}-\frac{x^{2}}{2} \ln (2 x),
$$

and, for $x \in\left[\frac{1}{2}, 1\right]$ :

$$
\Psi_{\alpha}(x)=x-\frac{1}{2}+\Psi_{\alpha}(1-x)
$$

and finally $\Psi_{\alpha}(x)=\Psi_{\alpha}(-x)$ for $x \in[-1,0]$, since $\psi_{\alpha}$ is an odd function. We are now able to calculate $\widehat{C}_{\alpha}^{+}\left(u_{1}, u_{2}\right)=\int_{0}^{u_{2}} \int_{0}^{u_{1}} \widehat{c}_{\alpha}\left(x_{1}-x_{2}\right) d x_{1} d x_{2}=\int_{0}^{u_{2}}\left(\psi_{\alpha}\left(u_{1}-x_{2}\right)-\right.$ $\left.\psi_{\alpha}\left(-x_{2}\right)\right) d x_{2}=\int_{0}^{u_{2}}\left(\psi_{\alpha}\left(u_{1}-x_{2}\right)+\psi_{\alpha}\left(x_{2}\right)\right) d x_{2}$ and so we get, in agreement with our earlier general result (2):

$$
\widehat{C}_{\alpha}^{+}\left(u_{1}, u_{2}\right)=\Psi_{\alpha}\left(u_{1}\right)+\Psi_{\alpha}\left(u_{2}\right)-\Psi_{\alpha}\left(u_{1}-u_{2}\right)
$$

The copula $\widehat{C}_{\alpha}^{-}$can then be calculated easily, since $C_{\gamma}^{-}\left(u_{1}, u_{2}\right)=u_{2}-C_{\gamma}^{+}\left(1-u_{1}, u_{2}\right)$ and therefore $\widehat{C}_{\alpha}^{-}\left(u_{1}, u_{2}\right)=\int_{0}^{\frac{1}{2}}\left(u_{2}-C_{\gamma}^{-}\left(1-u_{1}, u_{2}\right)\right) p_{\alpha}(\gamma) d \gamma=u_{2}-\widehat{C}_{\alpha}^{+}\left(1-u_{1}, u_{2}\right)$. We can also easily calculate the Spearman's rho of $\widehat{C}_{\alpha}^{+}$, since $\rho\left(\widehat{C}_{\alpha}^{+}\right)=\int_{0}^{1 / 2}(2 \gamma-1)(\gamma-$ 1) $2^{1 / \alpha} \gamma^{(1-\alpha) / \alpha} / \alpha d \gamma$ so that

$$
\rho\left(\widehat{C}_{\alpha}^{+}\right)=1-\frac{3}{2(1+\alpha)}+\frac{1}{2(1+2 \alpha)}
$$

and we have $\rho\left(\widehat{C}_{\alpha}^{-}\right)=-\rho\left(\widehat{C}_{\alpha}^{+}\right)$(this is a general relation between the rho of the periodic copulas with density $\tilde{c}\left(x_{1}+x_{2}\right)$ and $\left.\tilde{c}\left(x_{1}-x_{2}\right)\right)$. We can sum up in Table 1 the values for which a limit copula is reached. The families built previously are

\begin{tabular}{|c|c|c|c|}
\hline$\alpha$ & $C_{F}^{-}$ & $C^{\perp}$ & $C_{F}^{+}$ \\
\hline$C_{\alpha}^{+}$ & $/$ & $\frac{1}{2}$ & 0 \\
$C_{\alpha}^{-}$ & 0 & $\frac{1}{2}$ & $/$ \\
$\widehat{C}_{\alpha}^{+}$ & $/$ & 0 & $+\infty$ \\
$\widehat{C}_{\alpha}^{-}$ & $+\infty$ & 0 & $/$ \\
\hline
\end{tabular}

Table 1: Limit copulas for the parameterization $C_{\alpha}$ and $\widehat{C}_{\alpha}$ 
exchangeable, since the related $\tilde{c}$ are expectations of functions leading to par double primitives and therefore lead themselves to par double primitives, so that (2) yields symmetry.

However, we can also construct a smooth family of non symmetric copulas by defining $\tilde{c}$ as the expectation of the previous non symmetric function (6) with a random $\gamma$.

Indeed, if $Z \sim q$ where $q$ is a positive density of a probability measure on $[0,1]$, then $\bar{c}_{q}:=E\left[\bar{c}_{Z}\right]=\int_{0}^{1} \bar{c}_{\gamma}(x) q(\gamma) d \gamma$ is a positive 1-periodic function. Thus, as before, if we have a family of random variables $\left(Z_{\alpha}\right)_{\alpha \geq 0}$ concentrated on $[0,1]$ with densities $\left\{q_{\alpha}, \alpha \in\right] 0,+\infty[\}$ on $[0,1]$ and such that $Z_{\alpha} \underset{\alpha \rightarrow 0}{\stackrel{\mathcal{L}}{\longrightarrow}} 1$ and $Z_{\alpha} \underset{\alpha \rightarrow \infty}{\stackrel{\mathcal{L}}{\longrightarrow}} 0$, we can define

$$
\widehat{\bar{C}}_{\alpha}^{+}:=E\left[C_{Z_{\alpha}}^{+}\right]=\int_{0}^{1} C_{\gamma}^{+} q_{\alpha}(\gamma) d \gamma, \quad \widehat{\bar{C}}_{\alpha}^{-}:=E\left[C_{Z_{\alpha}}^{-}\right]=\int_{0}^{1} C_{\gamma}^{-} q_{\alpha}(\gamma) d \gamma
$$

(that correspond respectively to the periodic densities $\tilde{c}\left(x_{1}-x_{2}\right)=\bar{c}_{q_{\alpha}}\left(x_{1}-x_{2}\right)$ and $\left.\tilde{c}\left(x_{1}+x_{2}\right)=\bar{c}_{q_{\alpha}}\left(x_{1}+x_{2}\right)=E\left[\bar{c}_{Z_{\alpha}}\left(x_{1}+x_{2}\right)\right]\right)$.

We take $Z_{\alpha}:=U^{\alpha}$, where $U$ is a uniform random variable on $[0,1]$. Its density is

$$
q_{\alpha}(u)=\frac{1}{\alpha} u^{\frac{1-\alpha}{\alpha}}
$$

so that the associated periodic function $\tilde{c}=\bar{c}_{\alpha}$ is given in $[0,1]$ by

$$
\widehat{\bar{c}}_{\alpha}(x):=\bar{c}_{q_{\alpha}}=\frac{1}{1-\alpha}\left[1-x^{\frac{1-\alpha}{\alpha}}\right], \alpha \neq 1, \quad \widehat{\bar{c}}_{1}(x):=\bar{c}_{q_{1}}=-\ln (x) .
$$

In order to find an expression for the copula, we compute the primitive $g_{\alpha}$ of $\widehat{\bar{c}}_{\alpha}$ that vanishes at $x=0$. We have, for $x \in[0,1]$ :

$$
g_{\alpha}(x)=\frac{1}{1-\alpha}\left[x-\alpha x^{\frac{1}{\alpha}}\right], \alpha \neq 1, \quad g_{1}(x)=x-x \ln (x),
$$

and for $x \in[-1,0]$ we have $g_{\alpha}(x)=g_{\alpha}(1+x)-1$. Denote by $G_{\alpha}$ the primitive of $g_{\alpha}$ vanishing at 0 . For $x \in[0,1] G_{\alpha}$ is given by

$$
G_{\alpha}(x)=\frac{1}{1-\alpha}\left[\frac{1}{2} x^{2}-\frac{\alpha^{2}}{1+\alpha} x^{\frac{1+\alpha}{\alpha}}\right], \alpha \neq 1, \quad G_{1}(x)=\frac{3}{4} x^{2}-\frac{1}{2} x^{2} \ln (x),
$$

whereas for $x \in[-1,0]$ we have

$$
G_{\alpha}(x)=G_{\alpha}(1+x)-x-G_{\alpha}(1)
$$

By Fubini's theorem, the copulas defined by (9) are the same as the copulas associated with the periodic functions $\tilde{c}=\widehat{\bar{c}}_{\alpha}$ and defined by (2). We thus have, by (2) and using 
periodicity of $\widehat{\bar{c}}$ (thus replacing $\widehat{\bar{c}}(\cdot)$ by $\widehat{\bar{c}}(\cdot-1)$, which is helpful in some computational respects):

$$
\begin{aligned}
& \widehat{\bar{C}}_{\alpha}^{+}\left(u_{1}, u_{2}\right)=G_{\alpha}\left(u_{1}\right)+G_{\alpha}\left(-u_{2}\right)-G_{\alpha}\left(u_{1}-u_{2}\right) \\
& \widehat{\bar{C}}_{\alpha}^{-}\left(u_{1}, u_{2}\right)=G_{\alpha}\left(u_{1}+u_{2}-1\right)-G_{\alpha}\left(u_{2}-1\right)-G_{\alpha}\left(u_{1}-1\right)+G_{\alpha}(-1) .
\end{aligned}
$$

Calculating the related Spearman rho we find:

$$
\rho\left(\widehat{\bar{C}}_{\alpha}^{+}\right)=1-\frac{3}{1+\alpha}+\frac{2}{1+2 \alpha}
$$

and $\rho\left(\widehat{\bar{C}}_{\alpha}^{-}\right)=-\rho\left(\widehat{\bar{C}}_{\alpha}^{+}\right)$. As we know from $(2), \widehat{\bar{C}}_{\alpha}^{-}$, contrary to $\widehat{\bar{C}}_{\alpha}^{+}$, is a family of symmetric copulas, but this family is however interesting because it completes "naturally" the family $\widehat{\bar{C}}_{\alpha}^{+}$. In Table 2 we sum up the values of the parameter $\alpha$ for which the $\widehat{\bar{C}}$ copulas reach the limit copulas.

\begin{tabular}{|c|c|c|c|}
\hline & $C_{F}^{-}$ & $C^{\perp}$ & $C_{F}^{+}$ \\
\hline $\bar{C}_{\alpha}^{+}$ & $/$ & 1 & 0 \\
$\bar{C}_{\alpha}^{-}$ & 0 & 1 & $/$ \\
$\widehat{\bar{C}}_{\alpha}^{+}$ & $/$ & 0 & $+\infty$ \\
$\widehat{\bar{C}}_{\alpha}^{-}$ & $+\infty$ & 0 & $/$ \\
\hline
\end{tabular}

Table 2: Limit copulas for the parameterization $\bar{C}_{\alpha}$ and $\widehat{\bar{C}}_{\alpha}$

\subsection{Beyond the bivariate case}

There are several ways to extend the previous construction to build a copula in dimension $n>2$. In dimension $n>2$, we see that if $\tilde{c}$ is a 1-periodic function such that $\int_{0}^{1} \tilde{c}(x) d x=1$, then $\tilde{c}\left(\sum_{i=1}^{n} \varepsilon_{i} x_{i}\right)$ are densities of copulas when $\varepsilon_{i} \in\{-1,1\}$. However, these copulas cannot be obtained directly in terms of bivariate copulas and therefore require cumbersome calculations. In order to keep the analysis simple, we work instead with the densities already defined for the bivariate case. Consider the following proposition.

Proposition 2.1. Assume that $c_{1}, \ldots, c_{n-1}$ are densities of two dimensional copulas built through periodic densities $\tilde{c}_{1}, \ldots, \tilde{c}_{n-1}$, i.e. $c_{j}(x, y)=\tilde{c}_{j}\left(x+\varepsilon_{j} y\right)$ with $\varepsilon_{j} \in$ 
$\{-1,1\}$ and $\tilde{c}_{j}$ a nonnegative 1-periodic function with unit integral on a period. Set $\check{c}:=\left(c_{1}, \ldots, c_{n-1}\right)$. Then

$$
\begin{aligned}
& \check{C}_{1}\left(u_{1}, . ., u_{n}\right):=\int_{0}^{u_{1}} . . \int_{0}^{u_{n}} c_{1}\left(x_{1}, x_{2}\right) c_{2}\left(x_{2}, x_{3}\right) . . c_{n-1}\left(x_{n-1}, x_{n}\right) d x_{1} \ldots d x_{n} \\
& \check{C}_{2}\left(u_{1}, . ., u_{n}\right):=\int_{0}^{u_{1}} . . \int_{0}^{u_{n}} c_{1}\left(x_{1}, x_{2}\right) c_{2}\left(x_{1}, x_{3}\right) . . c_{n-1}\left(x_{1}, x_{n}\right) d x_{1} \ldots d x_{n}
\end{aligned}
$$

are copulas.

The proof is quite immediate. Properties 1 and 3 in Section 1 are satisfied by construction. It remains to observe that $\check{C}\left(1, . ., 1, u_{k}, 1, . ., 1\right)=\int_{0}^{u_{k}} d x_{k}=u_{k}$, by using Fubini's theorem, integrating first with respect to the $x_{i}$ 's with $i \neq k$, and using then the property $\int_{x}^{1+x} \tilde{c}(u) d u=1$.

The first copula $\check{C}_{1}$ is convenient if we wish to express the $n$-dependence in terms of dependences of two consecutive variables, whereas the second one $\check{C}_{2}$ allows us to express the $n$-dependence in terms of the dependence of a preferred variable (the first in our formulation) with all other variables. The second method could be referred to as a "preferred-" or "main-factor" approach.

\section{The simulation of periodic copulas}

Let us begin by recalling how to simulate a copula that admits a density $p\left(x_{1}, \ldots, x_{n}\right)$. We need simulate a vector of uniform variables $\left(U_{1}, \ldots, U_{n}\right)$ that has the following joint cdf:

$$
C\left(u_{1}, \ldots, u_{n}\right)=\int_{0}^{u_{1}} . . \int_{0}^{u_{n}} p\left(x_{1}, \ldots, x_{n}\right) d x_{1} \ldots d x_{n} .
$$

This can be done according to the following steps.

- To simulate the first variable $U_{1}$, it suffices to sample from a uniform random variable $\tilde{U}_{1}$ in $[0,1]$. This can be easily done on a PC. Let us call $u_{1}$ the simulated sample.

- To obtain a sample $u_{2}$ from $U_{2}$ consistently with the earlier sampled $u_{1}$, we need to know the law of $U_{2}$ conditional on $U_{1}=u_{1}$. Let us name $F_{2}\left(. \mid u_{1}\right)$ the cdf of this law,

$$
\begin{aligned}
F_{2}\left(u_{2} \mid u_{1}\right) & =\mathbb{P}\left(U_{2} \leq u_{2} \mid U_{1}=u_{1}\right)=\partial_{u_{1}} C\left(u_{1}, u_{2}, 1, . ., 1\right) / \partial_{u_{1}} C\left(u_{1}, 1,1, . ., 1\right) \\
& =\partial_{u_{1}} C\left(u_{1}, u_{2}, 1, . ., 1\right)=\int_{0}^{u_{2}} \int_{0}^{1} \ldots \int_{0}^{1} p\left(u_{1}, x_{2}, . . x_{m}\right) d x_{2} . . d x_{m}
\end{aligned}
$$


We take $u_{2}=F_{2}^{-1}\left(\tilde{U}_{2} \mid u_{1}\right)$ where $\tilde{U}_{2}$ is a new uniform- $[0,1]$ sample independent of $\tilde{U}_{1}$.

- to simulate $U_{k}$ consistently with the earlier sampled $u_{1}, \ldots, u_{k-1}$, we need the law of $U_{k}$ conditional on $U_{i}=u_{i}$ for $i<k$. Denoting as usual by $F_{k}\left(\cdot \mid u_{1}, . ., u_{k-1}\right)$ the cdf of this law,

$$
\begin{aligned}
F_{k}\left(u_{k} \mid u_{1}, \ldots, u_{k-1}\right) & =\mathbb{P}\left(U_{k} \leq u_{k} \mid U_{1}=u_{1}, . ., U_{k-1}=u_{k-1}\right) \\
& =\frac{\partial_{u_{1}, . ., u_{k-1}} C\left(u_{1}, . ., u_{k}, 1, . ., 1\right)}{\partial_{u_{1}, . ., u_{k-1}} C\left(u_{1}, . ., u_{k-1}, 1, . ., 1\right)} \\
& =\frac{\int_{0}^{u_{k}} \int_{0}^{1} . . \int_{0}^{1} p\left(u_{1}, . ., u_{k-1}, x_{k}, . ., x_{n}\right) d x_{k} d x_{k+1} . . d x_{n}}{\int_{0}^{1} \int_{0}^{1} . . \int_{0}^{1} p\left(u_{1}, . ., u_{k-1}, x_{k}, . ., x_{n}\right) d x_{k} d x_{k+1} . . d x_{n}}
\end{aligned}
$$

we can take $U_{k}=F_{k}^{-1}\left(\tilde{U}_{k} \mid u_{1}, . ., u_{k-1}\right)$ where $\tilde{U}_{k}$ is a uniform-[0,1] variable independent of $\left(\tilde{U}_{1}, \ldots, \tilde{U}_{k-1}\right)$.

In the case of the periodic copulas $\check{C}_{1,2}$, maintaining the notation of Proposition 2.1, we have respectively $F_{k}^{1}\left(u_{k} \mid u_{1}, . ., u_{k-1}\right)=\int_{0}^{u_{k}} c_{k-1}\left(u_{k-1}, x_{k}\right) d x_{k}$ and $F_{k}^{2}\left(u_{k} \mid u_{1}, . ., u_{k-1}\right)=$ $\int_{0}^{u_{k}} c_{k-1}\left(u_{1}, x_{k}\right) d x_{k}$, where the upper index refers to the copula we are considering. Taking the smooth families of the previous section, these $F$ functions can be expressed in terms of $\psi_{\alpha}$ and $g_{\alpha}$ (for example $\int_{0}^{u_{k}} \widehat{c}_{\alpha}(u \pm x) d x= \pm\left(\psi_{\alpha}\left(u \pm u_{k}\right)-\psi_{\alpha}(u)\right)$. Moreover they are strictly increasing, and can therefore be inverted easily numerically. We note here that if we choose the "non smooth" copulas $C^{ \pm}$and $\bar{C}^{ \pm}$, this inversion is not feasible since the densities vanish on some intervals. Thus we have obtained families of $n$-dimensional copulas essentially characterized by $n-1$ parameters $\alpha_{i}$ plus the flags $s g n_{i}, s y m_{i}$, for $i=1, \ldots, n-1$, where $s y m_{i}$ is set according to whether we take a symmetric family or not (symbolized here by the $\overline{b a r}$ ), and where $s g n_{i}$ is taken from the set $\{-,+\}$.

\section{Conclusions}

The new family of "periodic" copulas introduced in this paper is an attempt at obtaining practically manageable and possibly asymmetric copulas. We have studied the two-dimensional case, based on a single dependence parameter, and then provided a means to construct an $n$-dimensional copula building on the two-dimensional case. We obtained families of copulas in dimension $n$ and parameterized by $n-1$ 
parameters, implying possibly asymmetric relations. We explain how such copulas can be simulated.

\section{References}

[1] Alfonsi A. (2002). Construction of copulas with periodic densities, working paper.

[2] Bouyé E., Durrleman V., Nikeghbali A., Riboulet G., Roncalli T. (2000). Copulas for Finance, A Reading Guide and Some Applications.

[3] Cherubini, U., and Luciano, E. (2002). Bivariate option pricing with copulas. Applied Mathematical Finance. 9 (2), 2002, pp. 69-86.

[4] Embrechts P., Lindskog F., McNeil A. (2001). Modelling Dependence with Copulas and Applications to Risk Management.

[5] Genz, A. and Bretz, F. (2002) Methods for the computation of multivariate t-probabilities. Journal of Computational and Graphical Statistics, 11, 950-971.

[6] Hürlimann, W. (2002). Hutchinson-Lai's conjecture for bivariate extreme value copulas. Statistics and Probability Letters 61(2), 191-198.

[7] Hürlimann, W. (2003). Fitting bivariate cumulative returns with copulas. Computational Statistics and Data Analysis, forthcoming.

[8] Joe, H. (1997). Multivariate Models and Dependence Concepts. Chapman \& Hall, London.

[9] Jouanin J.-F., Rapuch G., Riboulet G., Roncalli T. (2001), Modelling dependence for credit derivatives with copulas, Groupe de Recherche Oprationnelle, Crédit Lyonnais, France.

[10] Juri, A., and Wüthrich, M.V. (2002). Copula convergence theorems for tail events. Insurance Mathematics and Economics 30(3), 405-420.

[11] Klugman, S.A., and Parsa, R. (1999). Fitting bivariate loss distributions with copulas. Insurance Mathematics and Economics 24(1-2), 139-148.

[12] Nelsen, R. (1999). An Introduction to Copulas. Springer, New York. 
[13] Nelsen, R., Quesada-Molina J.J., Rodrguez-Lallena J. A., and beda-Flores M. (2001). Distribution functions of copulas: a class of bivariate probability integral transforms. Statistics and Probability Letters 54(3), 277-282.

[14] Prampolini, A. (2003). Modelling dependent defaults for the pricing and risk management of CDO's and basket credit derivatives. Electronic proceedings of the Risk Europe conference, Paris, April 8-9, 2003.

[15] Schönbucher, P., and Schubert, D. (2001). Copula-Dependent Default Risk in Intensity Models, working paper.

[16] Wei, G. and Hu, T. (2002). Supermodular dependence ordering on a class of multivariate copulas. Statistics and Probability Letters 57(4), 375-385 